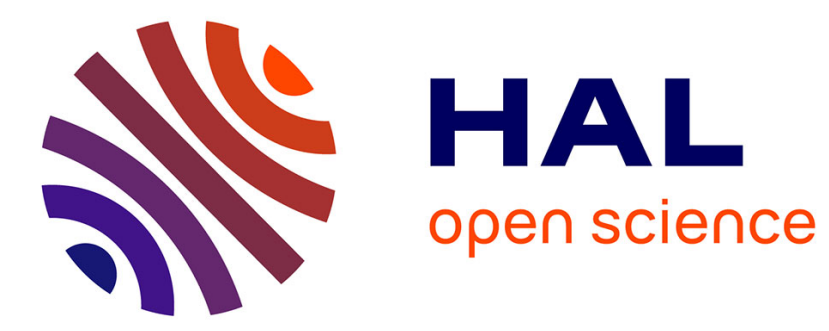

\title{
Estimation spatio-temporelle d'un modèle de système de particules
}

Xavier Guyon, Besnik Pumo

\section{To cite this version:}

Xavier Guyon, Besnik Pumo. Estimation spatio-temporelle d'un modèle de système de particules. Compte Rendu à l'Académie des Sciences, Série I, 2005, Série I (340), pp.619-622. 10.1016/j.crma.2005.03.014 . hal-00271931

\section{HAL Id: hal-00271931 \\ https://hal.science/hal-00271931}

Submitted on 10 Apr 2008

HAL is a multi-disciplinary open access archive for the deposit and dissemination of scientific research documents, whether they are published or not. The documents may come from teaching and research institutions in France or abroad, or from public or private research centers.
L'archive ouverte pluridisciplinaire $\mathbf{H A L}$, est destinée au dépôt et à la diffusion de documents scientifiques de niveau recherche, publiés ou non, émanant des établissements d'enseignement et de recherche français ou étrangers, des laboratoires publics ou privés. 


\title{
Estimation de Yule-Walker d'un CAR(p) observé à temps discret
}

\author{
Sandie SOUCHET \& Xavier GUYON
}

\section{Résumé}

Soit $(X(0), X(\delta), \cdots, X(n \delta))$ une observation discrète à pas $\delta>0$, petit mais fixé, d'un $\mathrm{CAR}(\mathrm{p})$ dont les dérivées ne sont pas observées. Pour estimer les paramètres de $X$, nous nous basons sur une estimation empirique des équations de Yule-Walker en 0. Cette estimation est asymptotiquement biaisée et doit être corrigée pour obtenir la convergence lorsque $T=n \delta \rightarrow$ $+\infty$. Une autre solution consiste à estimer les équations de Yule-Walker non plus en 0 mais en $-(p-1) \delta$. Cette deuxième méthode fournit directement des estimateurs convergeant, avec un biais d'ordre principal $\delta$ qui est explicite. Dans les deux cas, les estimateurs obtenus sont asymptotiquement normaux à la vitesse $\sqrt{T}$ et efficaces en variance. Le coefficient de diffusion est également estimé et l'estimateur proposé est asymptotiquement normal à la vitesse $\sqrt{T \delta^{-1}}$.

\section{Abstract}

Let be $(X(0), X(\delta), \cdots, X(n \delta))$ a discrete observation, with a fixed and small mesh $\delta>0$, of a $\operatorname{CAR}(\mathrm{p})$ with unobserved derivatives. In order to estimate the parameters of $X$, we use an estimation of Yule-Walker equations at point 0 . This estimation is asymptotically biased and must be corrected to converge as $T=n \delta \rightarrow+\infty$. An alternative method consists to estimating Yule-Walker equations at point $-(p-1) \delta$. This second method directly provides convergent estimators with a bias of main order $\delta$ which is explicit. In both cases, estimators are asymptotically normal with a rate in $\sqrt{T}$ and efficiency in variance. The diffusion coefficient is also estimated with a precision of order $\delta$ and the estimator is asymptotically normal with a rate in $\sqrt{T \delta^{-1}}$.

\section{Mots clefs}

Modèle autorégressif continu d'ordre $p$; équations de Yule-Walker; biais d'estimation; fonction d'autocovariance; fonctions de covariance dérivée.

Code A.M.S. 62 M 10 - 62 F 12.

\section{Introduction :}

Soit $W=(W(t))_{t>0}$ un mouvement brownien réel issu de 0 défini sur un espace de probabilité $(\Omega, \mathcal{A}, \mathbb{P})$. Un $\operatorname{CAR}(\mathrm{p})$ de paramètre $\theta=(\alpha, \sigma), \alpha={ }^{t}\left(\alpha_{0}, \cdots, \alpha_{p-1}\right)$, est un processus $X=(X(t))_{t \geq 0}$ dérivable à l'ordre $p-1$ sur $\mathbb{R}^{+}$tel que, si l'on note $X^{(j)}(t)=\frac{d}{d t^{j}} X(t)$, on a $([4],[7]):$

$$
d X^{(p-1)}(t)+\left[\alpha_{0} X(t)+\cdots+\alpha_{p-1} X^{(p-1)}(t)\right] d t=\sigma d W(t)
$$


Soit $P$ le polynôme caractéristique associé à $(1): P(z)=z^{p}+\alpha_{p-1} z^{p-1}+\cdots+\alpha_{0}$. Si toutes les racines de $P$ ont une partie réelle négative, il existe une unique diffusion gaussienne stationnaire et ergodique $Y={ }^{t}\left(X, X^{(1)}, \cdots, X^{(p-1)}\right)$ qui satisfait (1) [1]. Dans toute la suite, nous supposerons que cette condition est vérifiée.

Comme pour les modèles autorégressifs $\mathrm{AR}(\mathrm{p})$ à temps discret, les paramètres d'un $\mathrm{CAR}(\mathrm{p})$ peuvent être estimés à partir des équations de Yule-Walker. Dans le cas continu, ces équations sont basées sur les fonctions de covariance dérivée (DCVF) $\left(D_{j, k}\right)$ définies pour $i, j \in\{0,1, \cdots, p-1\}$ par (cf. [7]) :

$$
D_{i, j}(h)=\mathbb{E}\left[X^{(i)}(h) X^{(j)}(0)\right], \quad D_{i, p}(h) \stackrel{L^{2}(\mathbb{P})}{=} \lim _{T \rightarrow+\infty} \frac{1}{T} \int_{0}^{T} X^{(i)}(t+h) d X^{(p-1)}(t)
$$

avec ([7], théorème 3.1$)$ :

$$
D_{p-1, p}(0)=-\frac{\sigma^{2}}{2}
$$

Les équations de Yule-Walker décalées de $h$, avec $h \leq 0$, sont obtenues en multipliant l'équation (1) par $X^{(j)}(t+h)$ et en prenant l'espérance sous la loi stationnaire. Ainsi, pour $h \leq 0$, ces équations sont :

$$
D_{j, p}(h)+\alpha_{p-1} D_{j, p-1}(h)+\cdots+\alpha_{0} D_{j, 0}(h)=0, j=0, \cdots, p-1
$$

Si on note $\Gamma(h)=\left(D_{j, k}(h)\right)_{j, k=0, \cdots, p-1}$ et $\gamma(h)={ }^{t}\left(D_{0, p}(h), \cdots, D_{p-1, p}(h)\right), \alpha$ satisfait le système linéaire suivant :

$$
\gamma(h)+\Gamma(h) \alpha=0
$$

Une estimation des $\left(D_{j, k}\right)$ permet donc d'estimer $\alpha$. Dans [7], Hyndman estime (4) en $h=0$ en se basant sur une observation continue de $X$ et de ses $p-1$ dérivées sur $[0, T]$, c'està-dire lorsque la diffusion multidimensionnelle $Y$ est complétement observée. L'estimateur de $\alpha$ obtenu est consistant, asymptotiquement normal et efficace lorsque $T \rightarrow+\infty$. Nous considérons ici le cas où la seule donnée est l'observation discrétisée $(X(0), X(\delta), \cdots, X(n \delta))$ de $X$ sur [0,T], $n \delta=T$ avec $\delta>0$ fixé et $T \rightarrow+\infty$. Les dérivées de $X$ n'étant pas observées, elles doivent être approximées afin de pouvoir définir des estimateurs des DCVF en 0.

Dans le $\S 2$, nous rappelons quelques résultats préliminaires sur les DCVF et leur relation avec la fonction d'autocovariance de $X$.

Nous définissons au $\S 3$ des approximations des dérivées de $X$ et les estimateurs des DCVF en 0 qui en sont déduits. La corrélation entre les approximations de $X^{(p-1)}(t)$ et de $d X^{(p-1)}(t)$ engendre un biais multiplicatif systématique dans l'estimation de $D_{p-1, p}(0)$. Ce biais étant explicite en $p$ et indépendant des paramètres à estimer, il suffit de renormaliser l'estimateur concerné pour débiaiser la procédure. On obtient ainsi un système d'équations de Yule-Walker estimées en $h=0$ qui conduit à une estimation convergente de $\alpha$ à $\delta$ près, asymptotiquement normale à la vitesse $\sqrt{T}$ et efficace en variance à un terme $(I+o(1))$ près. L'estimateur du coefficient de diffusion $\sigma^{2}$ est convergent à un $(1+O(\delta))$ près et asymptotiquement normal à la vitesse $\sqrt{T \delta^{-1}}$.

Dans le $\S 4$, nous étudions une autre procédure qui consiste à estimer les équations de YuleWalker (4) non pas en $h=0$ mais décalées de $h=-(p-1) \delta$. Ce décalage élimine le biais systématique de l'estimation de $D_{p-1, p}$. Là encore, l'estimateur proposé est asymptotiquement normal à la vitesse $\sqrt{T}$, efficace, avec un biais dont la partie principale en $\delta$ est explicite.

Ces résultats sont mis en oeuvre pour un $\operatorname{CAR}(2)$ au $\S 5$. 


\section{Quelques propriétés de la fonction d'autocovariance}

Dans la suite, la notation $j=0, p$ signifie $j \in\{0, \cdots, p\}$.

Les calculs de biais et de variances asymptotiques des méthodes d'estimation sont basés sur les propriétés de la fonction d'autocovariance (ACVF) de $X, r(h)=\mathbb{E}[X(t+h) X(t)]$.

L'ACVF est de classe $\mathcal{C}^{2 p-2}$ sur $\mathbb{R}$ et de classe $\mathcal{C}^{\infty}$ sur $\mathbb{R}^{*}$. De plus, pour tout $k \geq 2 p-1$, les limites à droite $r^{(k)}\left(0^{+}\right)$et à gauche $r^{(k)}\left(0^{-}\right)$de $r^{(k)}$ en 0 existent et sont finies, avec :

$$
r^{(2 p-1)}\left(0^{-}\right)=-r^{(2 p-1)}\left(0^{+}\right), \quad r^{(2 p)}\left(0^{-}\right)=r^{(2 p)}\left(0^{+}\right)
$$

Enfin, $r$ et ses dérivées successives sont à décroissance exponentielle.

Il existe un lien fonctionnel entre l'ACVF, $r$, et les DCVF, $\left(D_{i, j}\right)$ (cf. théorème 3.1, [7]) :

\section{Propriété 1}

$$
\begin{aligned}
\forall h \in \mathbb{R}, \forall i, j=0, p-1, & D_{i, j}(h)=(-1)^{j} r^{(i+j)}(h)=(-1)^{i} r^{(i+j)}(-h) \\
\forall h \neq 0, \forall i=0, p-1, & D_{i, p}(h)=(-1)^{p} r^{(i+p)}(h)=(-1)^{i} r^{(i+p)}(-h) \\
& D_{p-1, p}(0)=(-1)^{p} r^{(2 p-1)}\left(0^{-}\right)=(-1)^{p-1} r^{(2 p-1)}\left(0^{+}\right)
\end{aligned}
$$

Les équations de Yule-Walker peuvent donc être exprimées en fonction de l'ACVF et de ses dérivées. Ainsi, pour tout $i=0, p-1$, on a :

$$
\begin{array}{ll}
\forall h \geq 0, & r^{(i+p)}\left(h^{+}\right)+\alpha_{p-1} r^{(i+p-1)}(h)+\cdots+\alpha_{0} r^{(i)}(h)=0 \\
\forall h \leq 0, & (-1)^{p} r^{(i+p)}\left(h^{-}\right)+(-1)^{p-1} \alpha_{p-1} r^{(i+p-1)}(h)+\cdots+\alpha_{0} r^{(i)}(h)=0
\end{array}
$$

Le lemme suivant nous permettra de calculer les biais d'estimation.

\section{Lemme 1}

Pour tout $h \geq 0$ et $\forall t \in]-\infty,-h] \cup[0,+\infty[$, on $a$ :

$$
r(t+h)=\sum_{k=0}^{2 p} \frac{h^{k}}{k !} r^{(k)}\left(t^{+}\right)+h^{2 p+1} R(t)
$$

où $R$ décroit exponentiellement vers 0 lorsque $|t| \rightarrow+\infty$. Pour $h \geq 0$ et $t \in]-h, 0[$, on a :

$$
r(t+h)=\sum_{k=0}^{2 p} \frac{h^{k}}{k !} r^{(k)}(t)+2 \frac{(t+h)^{2 p-1}}{(2 p-1) !} r^{(2 p-1)}\left(0^{+}\right)+O\left(h^{2 p+1}\right)
$$

Ce résultat est une conséquence directe de l'application de la formule de Taylor avec reste intégral.

\section{Estimation des équations de Yule-Walker en $h=0$}

Les DCVF étant liées aux dérivées de $X$ qui ne sont pas observées, nous sommes amenés à définir des approximations de ces dérivées. Soit $\Delta$ l'opérateur de différence première, $\Delta X(t+\delta)=X(t+\delta)-X(t)$, et $\Delta^{(j)}$ son $j^{\text {ème }}$ itéré, $\Delta^{(j)} X(t+j \delta)=\sum_{l=0}^{j} C_{j}^{l}(-1)^{j-l} X(t+l \delta)$. Il est naturel d'approximer $X^{(j)}(t)$ par $\delta^{-j} \Delta^{(j)} X(t+j \delta)$. En effet, le théorème qui suit, 
établit que, pour $j=1, p, \delta^{-j} \Delta^{(j)} X(t+j \delta)$ approche $d X^{(j-1)}(t)$ à $\delta$ près.

On introduit, pour $(k, j)$ tels que $1 \leq k \leq j \leq p$ :

$$
f_{j, k}^{p}(t)=\sum_{l=k}^{j} C_{j}^{l}(-1)^{j-l} \frac{(t+l \delta)^{p-1}}{(p-1) !}
$$

Pour $j \in \mathbb{N}^{*}, k \in \mathbb{N}: a_{j}^{k}=\sum_{l=0}^{j} C_{j}^{l}(-1)^{j-l} l^{k} ;$ On vérifie que $: \forall k=0, j-1, a_{j}^{k}=0, a_{j}^{j}=j$ ! et $a_{j}^{j+1}=\frac{j}{2}(j+1)$ ! .

Avec $\beta_{0}=\alpha_{p-1} \alpha_{0}, i=1, p-1, \beta_{i}=\alpha_{p-1} \alpha_{i}-\alpha_{i-1}, \gamma_{0}=-\beta_{p-1} \alpha_{0}$ et $i=1, \cdots, p-1$, $\gamma_{i}=\beta_{i-1}-\beta_{p-1} \alpha_{i}$, on a :

\section{Théorème 1}

$$
\begin{aligned}
\Delta^{(j)} X(t+j \delta) & =\sum_{k=j}^{p-1} \frac{a_{j}^{k} \delta^{k}}{k !} X^{(k)}(t)-\frac{a_{j}^{p} \delta^{p}}{p !} \sum_{i=0}^{p-1} \alpha_{i} X^{(i)}(t)+\frac{a_{j}^{p+1} \delta^{p+1}}{(p+1) !} \sum_{i=0}^{p-1} \beta_{i} X^{(i)}(t)+R_{j}(t) \\
& +B_{j}^{p}(t)-\alpha_{p-1} B_{j}^{p}(t)+\beta_{p-1} B_{j}^{p+1}(t)
\end{aligned}
$$

où :

$B_{j}^{p}(t)=\sum_{k=1}^{j} \int_{t+(k-1) \delta}^{t+k \delta} f_{j, k}^{p}(t-v) \sigma d W(v) \quad$ et $R_{j}(t)=\sum_{k=1}^{j} \int_{t+(k-1) \delta}^{t+k \delta} f_{j, k}^{p+2}(t-v)\left(\sum_{i=0}^{p-1} \gamma_{i} X^{(i)}(v)\right) d v$

La démonstration de ce résultat est donnée en annexe $\mathrm{A}$.

Ainsi, pour $i=0, p-1$ et $j=0, p$, un estimateur empirique de $D_{i, j}(0)$ est :

$$
\widehat{D}_{i, j}^{n}=\frac{\delta^{-(i+j)}}{n+1-p} \sum_{k=0}^{n-p} \Delta^{(i)} X(k \delta+i \delta) \Delta^{(j)} X(k \delta+j \delta)
$$

Notons, pour $i, j \in \mathbb{N}: d_{i, j}=2 \sum_{l=0}^{i} C_{i}^{l}(-1)^{l} \frac{(j-l)^{2 p-1}}{(2 p-1) !}$. Les DCVF estimées et les dérivées de l'ACVF sont en correspondance comme suit :

\section{Théorème 2}

Pour $t \geq 0, i, j=0, p$ et $k \in \mathbb{Z}$, on $a:$

$$
\mathbb{E}\left[\Delta^{(i)} X(t+i \delta) \Delta^{(j)} X(t+j \delta+k \delta)\right]=(-1)^{i} \Delta^{(i+j)} r(j \delta+k \delta)=(-1)^{j} \Delta^{(i+j)} r(i \delta-k \delta)
$$

De plus, si $k \leq-j$ ou $k \geq i$, on $a$ :

$$
\Delta^{(i+j)} r(j \delta+k \delta)=\sum_{l=i+j}^{2 p} \frac{\delta^{l}}{l !} a_{i+j}^{l} r^{(l)}\left((k \delta-i \delta)^{+}\right)+\delta^{2 p+1} R(k \delta)
$$

où $R$ est à décroissance exponentielle. Enfin, si $k \in\{-j+1, \cdots, i-1\}$ alors :

$$
\Delta^{(i+j)} r(j \delta+k \delta)=\sum_{l=i+j}^{2 p} \frac{\delta^{l}}{l !} a_{i+j}^{l} r^{(l)}((k-i) \delta)+\delta^{2 p-1} d_{i+j, k+j} r^{(2 p-1)}\left(0^{+}\right)+O\left(\delta^{2 p+1}\right)
$$




\section{Démonstration:}

Notons : $A=\mathbb{E}\left[\Delta^{(i)} X(t+i \delta) \Delta^{(j)} X(t+j \delta+k \delta)\right]$. (10) repose sur la définition de $\Delta^{(i)}$ :

$$
\begin{aligned}
A & =\sum_{l=0}^{i} C_{i}^{l}(-1)^{i-l} \sum_{n=0}^{j} C_{j}^{n}(-1)^{n} \mathbb{E}[X(t+l \delta) X(t+k \delta+j \delta-n \delta)] \\
& =\sum_{l=0}^{i} C_{i}^{l}(-1)^{i-l} \sum_{n=0}^{j} C_{j}^{n}(-1)^{n} r(k \delta+j \delta-n \delta-l \delta) \\
& =(-1)^{i} \sum_{m=0}^{i+j}(-1)^{m}\left(\sum_{l=\max \{0, m-j\}}^{\min \{m, i\}} C_{i}^{l} C_{j}^{m-l}\right) r(k \delta+j \delta-m \delta) \\
& =(-1)^{i} \sum_{m=0}^{i+j}(-1)^{m} C_{i+j}^{m} r(k \delta+j \delta-m \delta)=(-1)^{i} \Delta^{(i+j)} r(k \delta+j \delta)
\end{aligned}
$$

Le membre de droite de l'égalité (10) est obtenue de manière similaire. Les équations (11) et (12) résultent d'une application directe du lemme 1.

Il est alors facile de montrer que les $\left(\widehat{D}_{i, j}^{n}\right)$ estiment les $\left(D_{i, j}(0)\right)$ à un $O(\delta)$ près mais que $\widehat{D}_{p-1, p}^{n}$ est biaisé. En effet, définissant $c(p)=-1+d_{2 p-1, p}$, on a :

\section{Proposition 1 : Biais des DCVF estimées en $h=0$}

Pour $i=0, p-1$ et $j=0, p$ tels que $i+j \leq 2 p-3$, on $a$ :

$$
\begin{aligned}
\mathbb{E}\left[\widehat{D}_{i, j}^{n}\right] & =(-1)^{i} r^{(i+j)}(0)+\delta\left(\frac{j-i}{2}\right)(-1)^{i} r^{(i+j+1)}(0)+O\left(\delta^{2}\right) \\
& =D_{i, j}(0)+\delta\left(\frac{j-i}{2}\right) D_{i, j+1}(0)+O\left(\delta^{2}\right)
\end{aligned}
$$

Pour $i=p-2$ et $j=p$ :

$$
\begin{aligned}
\mathbb{E}\left[\widehat{D}_{p-2, p}^{n}\right] & =(-1)^{p-2} r^{(2 p-2)}(0)+\delta\left(-1+d_{2 p-2, p}\right)(-1)^{p-2} r^{(2 p-1)}\left(0^{+}\right)+O\left(\delta^{2}\right) \\
& =D_{p-1, p-1}(0)-\delta\left(-1+d_{2 p-2, p}\right) D_{p-1, p}(0)+O\left(\delta^{2}\right)
\end{aligned}
$$

Pour $i=p-1$ et $j=p-1$ :

$$
\begin{aligned}
\mathbb{E}\left[\widehat{D}_{p-1, p-1}^{n}\right] & =(-1)^{p-1} r^{(2 p-2)}(0)+\delta d_{2 p-2, p-1}(-1)^{p-1} r^{(2 p-1)}\left(0^{+}\right)+O\left(\delta^{2}\right) \\
& =D_{p-1, p-1}(0)+\delta d_{2 p-2, p-1} D_{p-1, p}(0)+O\left(\delta^{2}\right)
\end{aligned}
$$

Pour $i=p-1$ et $j=p$ :

$$
\begin{aligned}
\mathbb{E}\left[\widehat{D}_{p-1, p}^{n}\right] & =c(p)(-1)^{p-1} r^{(2 p-1)}\left(0^{+}\right)+\frac{\delta}{2}(-1)^{p-1} r^{(2 p)}\left(0^{+}\right)+O\left(\delta^{2}\right) \\
& =c(p) D_{p-1, p}(0)-\frac{\delta}{2} \sum_{j=0}^{p-1} \alpha_{j} D_{p-1, j+1}(0)+O\left(\delta^{2}\right)
\end{aligned}
$$




\section{Démonstration :}

Utilisant (10) et (12), on a, pour $i=0, p-1, j=0, p$ et $i+j \leq 2 p-3$ :

$$
\begin{aligned}
\mathbb{E}\left[\widehat{D}_{i, j}^{n}\right] & =\delta^{-(i+j)} \mathbb{E}\left[\Delta^{(i)} X(i \delta) \Delta^{(j)} X(j \delta)\right]=\delta^{-(i+j)}(-1)^{i} \Delta^{(i+j)} r(j \delta) \\
& =(-1)^{i} \frac{a_{i+j}^{i+j}}{(i+j) !} r^{(i+j)}(-i \delta)+(-1)^{i} \delta \frac{a_{i+j}^{i+j+1}}{(i+j+1) !} r^{(i+j+1)}(-i \delta)+O\left(\delta^{2}\right) \\
& =(-1)^{i} r^{(i+j)}(-i \delta)+(-1)^{i} \delta\left(\frac{i+j}{2}\right) r^{(i+j+1)}(-i \delta)+O\left(\delta^{2}\right)
\end{aligned}
$$

Par la formule de Taylor : $r^{(i+j)}(0)=r^{(i+j)}(-i \delta)+i \delta r^{(i+j+1)}(-i \delta)+O\left(\delta^{2}\right)$.

$$
\begin{aligned}
\mathbb{E}\left[\widehat{D}_{i, j}^{n}\right] & =(-1)^{i} r^{(i+j)}(0)+(-1)^{i} \delta\left(\frac{j-i}{2}\right) r^{(i+j+1)}(-i \delta)+O\left(\delta^{2}\right) \\
& =(-1)^{i} r^{(i+j)}(0)+(-1)^{i} \delta\left(\frac{j-i}{2}\right) r^{(i+j+1)}(0)+O\left(\delta^{2}\right)
\end{aligned}
$$

D'après la propriété $1: D_{i, j}(0)=(-1)^{i} r^{(i+j)}(0)$ et $D_{i, j+1}(0)=(-1)^{i} r^{(i+j+1)}(0)$. On obtient le résultat annoncé.

Pour montrer (13), on utilise de même (10) et (12) avec $i=p-1, j=p$ et $k=0$, le développement de Taylor de $r^{(2 p-1)}$ et $r^{(2 p)}$ en $0^{-}$, puis la propriété 1 , on obtient :

$$
\begin{aligned}
& \mathbb{E}\left[\widehat{D}_{p-1, p}^{n}\right]=(-1)^{p-1} \delta^{-(2 p-1)} \Delta^{(2 p-1)} r(p \delta) \\
& =(-1)^{p-1}\left[r^{(2 p-1)}(-(p-1) \delta)+d_{2 p-1, p} r^{(2 p-1)}\left(0^{+}\right)+\delta\left(\frac{2 p-1}{2}\right) r^{(2 p)}(-(p-1) \delta)\right]+O\left(\delta^{2}\right) \\
& =(-1)^{p-1}\left[r^{(2 p-1)}\left(0^{-}\right)-(p-1) \delta r^{(2 p)}\left(0^{-}\right)+d_{2 p-1, p} r^{(2 p-1)}\left(0^{+}\right)+\delta\left(\frac{2 p-1}{2}\right) r^{(2 p)}\left(0^{-}\right)\right]+O\left(\delta^{2}\right) \\
& =(-1)^{p-1}\left(-1+d_{2 p-1, p}\right) r^{(2 p-1)}\left(0^{+}\right)+(-1)^{p-1} \delta\left(\frac{1}{2}\right) r^{(2 p)}\left(0^{+}\right)+O\left(\delta^{2}\right)
\end{aligned}
$$

En dérivant $(5)$ pour $i=p-1$ et en passant à la limite en $0^{+}$, on obtient : $r^{(2 p)}\left(0^{+}\right)=$ $-\sum_{j=0}^{p-1} \alpha_{j} r^{(j+p)}\left(0^{+}\right)$. Avec la propriété 1 , on a : $(-1)^{p-1} \sum_{j=0}^{p-1} \alpha_{j} r^{(j+p)}\left(0^{+}\right)=\sum_{j=0}^{p-1} \alpha_{j} D_{p-1, j+1}(0)$. Les autres développements s'obtiennent de manière similaire.

Comme le montre (13), $\widehat{D}_{p-1, p}^{n}$ présente un biais multiplicatif systématique. Le coefficient multiplicateur $c(p)$ est connu en fonction de $p$ et indépendant de $\left(\alpha, \sigma^{2}\right)$. On a, par exemple : $c(1)=1, c(2)=\frac{2}{3}, c(3)=\frac{11}{20}=0.55, c(4)=\frac{151}{315} \simeq 0.48$ et $c(5)=\frac{15619}{36288} \simeq 0.43$. Ainsi, en renormalisant $\widehat{D}_{p-1, p}^{n}$ par $c(p)$, on obtient un estimateur de $D_{p-1, p}(0)$ à un $O(\delta)$ près . D'après (2), il est naturel d'estimer $\sigma^{2}$ par $\widehat{\sigma}_{n}^{2}=-\frac{2}{c(p)} \widehat{D}_{p-1, p}^{n}$. Ce paramètre $\sigma^{2}$ peut aussi être estimé à partir de la variation quadratique de $\Delta^{(p-1)} X$, VQ $\left(\Delta^{(p-1)} X\right)=\sum_{k=0}^{n-p}\left[\Delta^{(p)} X(k \delta+p \delta)\right]^{2}$. Une application directe de (10) et (12) permet d'obtenir :

$$
\mathbb{E}\left[\operatorname{VQ}\left(\Delta^{(p-1)} X\right)\right]=(n-p+1) \delta^{2 p-1} c(p) \sigma^{2}(1+O(\delta))
$$

Concernant $\alpha, \Gamma(0)$ et $\gamma(0)$ sont estimés par :

$$
\widehat{\Gamma}^{n}=\left(\widehat{D}_{i, j}^{n}\right)_{i, j=0, p-1} \text { et } \widehat{\gamma}^{n}={ }^{t}\left(\widehat{D}_{0, p}^{n}, \cdots, \widehat{D}_{p-2, p}^{n}, c(p)^{-1} \widehat{D}_{p-1, p}^{n}\right)
$$


(4) est alors estimé par :

$$
\widehat{\Gamma}^{n} \widehat{\alpha}+\widehat{\gamma}^{n}=0
$$

Le résultat suivant montre que, pour $\delta$ proche de 0 et $n \rightarrow+\infty$, ce système admet une unique solution qui estime $\alpha$ avec un biais de l'ordre de $\delta$, biais qui s'explicite en fonction des quantités suivantes, pour $i=0, p-3$ :

$$
\begin{aligned}
& B_{i}=\frac{1}{2} \sum_{j=0}^{p-1} \alpha_{j}(j-p) D_{i, j+1}(0), B_{p-2}=\frac{1}{2} \sum_{j=0}^{p-1} \alpha_{j}(j-p) D_{p-2, j+1}(0)+\left(-1+\frac{1}{2} d_{2 p-2, p}\right) \sigma^{2} \\
& B_{p-1}=\frac{1}{2} \sum_{i=0}^{p-1} \alpha_{i}\left(i+1-p-c(p)^{-1}\right) D_{p-1, i+1}(0)-\frac{\alpha_{p-1}}{2} d_{2 p-2, p-1} \sigma^{2}
\end{aligned}
$$

Posons $\Gamma^{\delta}=\mathbb{E}\left[\widehat{\Gamma}^{n}\right], \gamma^{\delta}=\mathbb{E}\left[\widehat{\gamma}^{n}\right], \sigma_{\delta}^{2}=\mathbb{E}\left[\widehat{\sigma}_{n}^{2}\right]$. On a :

Théorème 3 : Convergence de l'estimation de Yule-Walker en $h=0$

Il existe $\delta_{0}>0$ tel que pour tout $\delta, 0<\delta \leq \delta_{0}$, avec une probabilité qui tend vers 1 lorsque $n \rightarrow+\infty$, le système (14) admet une unique solution $\widehat{\alpha}^{n}=-\left(\widehat{\Gamma}^{n}\right)^{-1} \widehat{\gamma}^{n}$.

De plus, $\widehat{\alpha}^{n} \stackrel{\mathbb{P}}{\rightarrow} \alpha^{\delta}=-\left(\Gamma^{\delta}\right)^{-1} \gamma^{\delta}$ avec $\alpha^{\delta}=\alpha-\delta \Gamma(0)^{-1} t\left(B_{0}, \cdots, B_{p-1}\right)+O\left(\delta^{2}\right)$.

De même, $\widehat{\sigma}_{n}^{2}$ converge en probabilité vers $\sigma_{\delta}^{2}=\sigma^{2}+\frac{\delta}{c(p)} \sum_{i=0}^{p-1} \alpha_{i} D_{p-1, i+1}(0)+O\left(\delta^{2}\right)$

Démonstration :

D'après la proposition $1, \Gamma^{\delta}=\Gamma(0)+O(\delta)$ et $\Gamma(0)$ est inversible ([7], proposition 3.1). Il existe donc $\delta_{0}>0$ tel que pour $\delta, 0<\delta \leq \delta_{0}, \Gamma^{\delta}$ est inversible. D'après [6] (§2.5.3, proposition 3), la diffusion $Y$ est $\alpha$-mélangeante avec un coefficient de mélange qui décroit exponentiellement. Les conditions requises pour appliquer la loi des grands nombres (mais aussi le théorème de la limite centrale) à $(X(k \delta))_{k \geq 0}$ sont donc satisfaites. Par application de la loi des grands nombres, $\widehat{\Gamma}^{n}$ converge en probabilité vers $\Gamma^{\delta}$. Ainsi, avec une probabilité qui tend vers 1 lorsque $n \rightarrow+\infty, \widehat{\Gamma}^{n}$ est inversible et (14) admet une unique solution $\widehat{\alpha}^{n}$. Appliquant à nouveau la loi des grands nombres à $\widehat{\gamma}^{n}$, on obtient la convergence de $\widehat{\alpha}^{n}$ vers $\alpha^{\delta}$. L'écart entre $\alpha^{\delta}$ et $\alpha$ est donné par :

$$
\alpha^{\delta}-\alpha=-\left(\Gamma^{\delta}\right)^{-1}\left[\gamma^{\delta}+\Gamma^{\delta} \alpha\right], \text { avec } \Gamma^{\delta}=\Gamma(0)+O(\delta)
$$

Utilisant la proposition 1 ainsi que les équations, (3) ou (5), on montre que :

$$
\gamma^{\delta}+\Gamma^{\delta} \alpha=\delta^{t}\left(B_{0}, \cdots, B_{p-1}\right)+O\left(\delta^{2}\right)
$$

On obtient ainsi le biais annoncé.

La convergence de $\widehat{\sigma}_{n}^{2}$ vers $\sigma_{\delta}^{2}$ est obtenue en appliquant la loi des grands nombres à $\widehat{D}_{p-1, p}^{n}$; l'évaluation du biais d'estimation résulte de (13).

Ces estimateurs sont asymtotiquement normaux, à la vitesse $\sqrt{T}$ pour $\widehat{\alpha}^{n}$, et $\sqrt{T \delta^{-1}}$ pour $\widehat{\sigma}_{n}^{2}$. $\widehat{\alpha}^{n}$ est efficace en variance à un facteur $(1+o(1))$ près puisque $\Gamma(0)$ est l'information de Fisher associée au modèle. Notons : $d(p)=\frac{2}{c(p)^{2}} \sum_{k=1}^{2 p-1}\left[\sum_{l=0}^{k} C_{2 p}^{l}(-1)^{l} \frac{(k-l)^{2 p-1}}{(2 p-1) !}\right]^{2}$ 
Théorème 4 : Normalité et efficacité de l'estimation de Yule-Walker en $h=0$

$$
\sqrt{n}\left(\widehat{\sigma}_{n}^{2}-\sigma_{\delta}^{2}\right) \stackrel{\mathcal{D}(\mathbb{P})}{\longrightarrow} \mathcal{N}\left(0, K_{\theta}\right), \quad K_{\theta}=d(p) \sigma^{4}+O(\delta)
$$

Pour tout $\delta, 0<\delta \leq \delta_{0}: \sqrt{n \delta}\left(\widehat{\alpha}^{n}-\alpha^{\delta}\right) \stackrel{\mathcal{D}(\mathbb{P})}{\rightarrow} \mathcal{N}_{p}\left(0, V_{\theta}\right), \quad V_{\theta}=\sigma^{2} \Gamma(0)^{-1}(I+o(1))$

La démonstration de ce résultat est donnée en annexe $\mathrm{B}$.

Nous allons proposer une procédure d'estimation de $\alpha$, directe, basée sur les équations de Yule-Walker avec un décalage de $h=-(p-1) \delta$.

\section{Estimation des équations de Yule-Walker décalées}

On sait qu'un $\operatorname{CAR}(\mathrm{p})$ observé aux temps discrets $k \delta$ est un $\operatorname{ARMA}(\mathrm{p}, \mathrm{p}-1)([2]$ [8]). Le théorème 1 précise cette correspondance. Avec $c(p)$ la constante définie en (13) et si on note $A$ l'opérateur, $A X(t)=\Delta^{(p)} X(t+p \delta)+\sum_{l=0}^{p-1} \alpha_{l} \delta^{p-l} \Delta^{(l)} X(t+l \delta)$, on obtient :

Corollaire 1 Si X est stationnaire :

$$
A X(k \delta)=B_{p}^{p}(k \delta)+R(k \delta), B_{p}(k \delta)=\sum_{i=1}^{p} \int_{k \delta+(i-1) \delta}^{k \delta+i \delta} f_{p, i}^{p}(k \delta-v) d W(v)
$$

$\left(B_{p}^{p}, R\right)$ est un processus gaussien, stationnaire, centré, vérifiant :

(i) $R$ est de variance $k(p) \sigma^{2} \delta^{2 p+1}$ et de covariance à décroissance exponentielle.

(ii) $B_{p}^{p}$ est de variance $c(p) \sigma^{2} \delta^{2 p-1}$. Sa corrélation $\rho$ est de portée $(p-1) \delta$, avec pour $k$ tel que $|k| \leq p-1$ :

$$
\rho(k)=c(p)^{-1} \sum_{i=1+k}^{p} \int_{i-1}^{i}\left(\sum_{l=i}^{p+k} C_{p}^{l-k}(-1)^{p+k-l} \frac{(l-u)^{p-1}}{(p-1) !}\right)\left(\sum_{j=i}^{p} C_{p}^{j}(-1)^{p-j} \frac{(j-u)^{p-1}}{(p-1) !}\right) d u
$$

Par exemple, pour un $\operatorname{CAR}(2), \rho(1)=\frac{1}{4}$; pour un $\operatorname{CAR}(3): \rho(1)=\frac{13}{33}, \rho(2)=\frac{1}{66}$; pour un $\operatorname{CAR}(4)$, on $\mathrm{a}: \rho(1)=\frac{1191}{2416}, \rho(2)=\frac{15}{302}, \rho(3)=\frac{1}{2416}$.

$B_{p}^{p}$ constitue ainsi la composante principale de la partie $M A(p-1)$. L'estimateur des moindres carrés $\bar{\alpha}^{n}$ associé à ce schéma satisfait, pour tout $i=0, p-1$ :

$$
\sum_{k=0}^{n-p} \Delta^{(i)} X(k \delta+i \delta)\left[\Delta^{(p)} X(k \delta+p \delta)+\sum_{j=0}^{p-1} \delta^{p-j} \Delta^{(j)} X(k \delta+j \delta) \bar{\alpha}_{j}^{n}\right]=0
$$

Ce système est celui des équations de Yule-Walker estimées en 0 avant le débiaisage de $\widehat{D}_{p-1, p}^{n}$ : le biais asymptotique s'explique par l'existence d'une corrélation entre le bruit $B_{p}^{p}(k \delta)$ et la variable explicative ${ }^{t}\left(X(k \delta), \cdots, \Delta^{(p-1)} X(k \delta+(p-1) \delta)\right)$. Dans un tel cas, une alternative classique aux moindres carrés est la méthode d'estimation par variable instrumentale : cette méthode ([3]) consiste à remplacer la variable explicative corrélée au bruit par un instrument 
qui en est décorrélé. $B_{p}^{p}(k \delta+(p-1) \delta)$ étant décorrélé de ${ }^{t}\left(X(k \delta), \cdots, \Delta^{(p-1)} X(k \delta+(p-1) \delta)\right)$, une solution consiste donc à estimer $\alpha$ par $\widetilde{\alpha}^{n}$ tel que, pour tout $i=0, p-1$ :

$$
\sum_{k=0}^{n+1-2 p} \Delta^{(i)} X(k \delta+i \delta)\left[\Delta^{(p)} X(k \delta+(2 p-1) \delta)+\sum_{j=0}^{p-1} \delta^{p-j} \Delta^{(j)} X(k \delta+(j+p-1) \delta) \widetilde{\alpha}_{j}^{n}\right]=0
$$

Notant, pour $i=0, p-1, j=0, p$ :

$$
\widetilde{D}_{i, j}^{n}=\frac{\delta^{-(i+j)}}{n+2-2 p} \sum_{k=0}^{n+1-2 p} \Delta^{(i)} X(k \delta+i \delta) \Delta^{(j)} X(k \delta+(j+p-1) \delta)
$$

$\widetilde{\Gamma}^{n}=\left(\widetilde{D}_{i, j}^{n}\right)_{i, j=0, p-1}$ et $\widetilde{\gamma}^{n}={ }^{t}\left(\widetilde{D}_{0, p}^{n}, \cdots, \widetilde{D}_{p-1, p}^{n}\right), \widetilde{\alpha}^{n}$ doit satisfaire le système :

$$
\widetilde{\Gamma}^{n} \widetilde{\alpha}^{n}+\widetilde{\gamma}^{n}=0
$$

Les $\left(\widetilde{D}_{i, j}^{n}\right)$ estiment les DCVF non plus en 0 mais en $-(p-1) \delta$, avec les contrôles des biais suivants :

Proposition 2 : Biais des DCVF estimées en $h=-(p-1) \delta$

Pour $i=0, p-1$ et $j=0, p, \mathbb{E}\left[\widetilde{D}_{i, j}^{n}\right]=D_{i, j}(-(p-1) \delta)+\delta \widetilde{B}_{i, j}+O\left(\delta^{2}\right)$ avec :

$$
\widetilde{B}_{i, j}=\frac{j-i}{2}(-1)^{i} r^{(i+j+1)}((p-1) \delta)
$$

La démonstration de ce résultat repose sur une application directe du théorème 2 .

Si elle existe, $\widetilde{\alpha}^{n}$ est la solution des équations de Yule-Walker estimées en $-(p-1) \delta$. On montre, comme précédemment :

\section{Théorème 5 : Estimateurs de Yule-Walker décalés}

Il existe $\delta_{0}>0$ tel que pour tout $\delta, 0<\delta \leq \delta_{0}$, avec une probabilité qui tend vers 1 lorsque $n \rightarrow+\infty$, le système (15) admet une unique solution $\widetilde{\alpha}^{n}=-\left(\widetilde{\Gamma}^{n}\right)^{-1} \widetilde{\gamma}^{n}$ telle que :

$$
\widetilde{\alpha}^{n} \stackrel{\mathbb{P}}{\longrightarrow} \widetilde{\alpha}^{\delta}=-\left(\widetilde{\Gamma}^{\delta}\right)^{-1} \widetilde{\gamma}^{\delta}, \quad \widetilde{\alpha}^{\delta}=\alpha-\delta \Gamma(0)^{-1 t}\left(\widetilde{B}_{0}, \cdots, \widetilde{B}_{p-1}\right)+O\left(\delta^{2}\right)
$$

où, pour $i=0, p-1, \widetilde{B}_{i}=\frac{1}{2} \sum_{j=0}^{p-1} \alpha_{j}(j-p) D_{i, j+1}(0)$. De plus :

$$
\sqrt{n \delta}\left(\widetilde{\alpha}^{n}-\widetilde{\alpha}^{\delta}\right) \stackrel{\mathcal{D}(\mathbb{P})}{\longrightarrow} \mathcal{N}_{p}\left(0, \widetilde{V}_{\theta}\right), \widetilde{V}_{\theta}=\sigma^{2} \Gamma(0)^{-1}[I+o(1)]
$$

\section{$5 \quad$ Etude expérimentale}

Ces deux méthodes d'estimation sont mises en oeuvre pour un CAR(2) de paramètres $\alpha_{0}=2$, $\alpha_{1}=3$ et $\sigma^{2}=1$. Les observations sont simulées à l'aide d'un schéma d'Euler à pas 0.0001 sur $[0, T]$ ceci, pour différents choix de $(n, \delta)$. Nous calculons la moyenne et la variance empirique sur $N=200$ réalisations des estimateurs présentés. La moyenne et la variance empiriques de l'estimation de $\alpha$ basée sur les équations de Yule-Walker en 0 sont notées respectivement : 
$m_{N}^{0}(\alpha)=\frac{1}{N} \sum_{i=1}^{N} \widehat{\alpha}^{n, i}$ et $V_{N}^{0}(\alpha)=\frac{1}{N-1} \sum_{i=1}^{N}\left(\widehat{\alpha}^{n, i}-m_{N}^{0}\right)^{2}$. Pour les équations de YuleWalker décalées, et pour $\sigma^{2}$, les notations sont : $m_{N}^{d}(\alpha), V_{N}^{d}(\alpha), m_{N}\left(\sigma^{2}\right), V_{N}\left(\sigma^{2}\right)$.

La fonction d'autocovariance d'un $\operatorname{CAR}(2)$ est, pour $a \neq b([5])$ :

$$
r(h)=\frac{\sigma^{2}}{2 a b\left(b^{2}-a^{2}\right)}(b \exp (-a|h|)-a \exp (-b|h|)), \quad a+b=\alpha_{1}, a b=\alpha_{0}
$$

Avec $\alpha_{0}=2, \alpha_{1}=3$ et $\sigma^{2}=1, r(h)=\frac{1}{6}(\exp (-|h|)-2 \exp (-2|h|))$. La variance théorique de $\widehat{\alpha}^{n}$ et $\widetilde{\alpha}^{n}$ est $\sigma^{2} \Gamma(0)^{-1}(I+o(1))$ où :

$$
\sigma^{2} \Gamma(0)^{-1}=\sigma^{2}\left(\begin{array}{cc}
r(0)^{-1} & 0 \\
0 & -r^{(2)}(0)^{-1}
\end{array}\right)=\left(\begin{array}{cc}
2 \alpha_{0} \alpha_{1} & 0 \\
0 & 2 \alpha_{1}
\end{array}\right)=\left(\begin{array}{cc}
12 & 0 \\
0 & 6
\end{array}\right)
$$

La variance théorique de $\widehat{\sigma}_{n}^{2}$ est : $\frac{9}{4} \sigma^{4}+O(\delta)$.

Les biais théoriques de $\widehat{\alpha}^{n}, \widetilde{\alpha}^{n}$ et $\widehat{\sigma}_{n}^{2}$ sont de la forme $B\left(\alpha, \sigma^{2}\right) \delta+O\left(\delta^{2}\right)$ avec respectivement :

$$
B^{0}(\alpha)=\left(\begin{array}{c}
-\frac{1}{2} \alpha_{0} \alpha_{1} \\
\frac{5}{4}\left(\alpha_{0}-\frac{1}{3} \alpha_{1}^{2}\right)
\end{array}\right), \quad B^{d}(\alpha)=\left(\begin{array}{c}
-\frac{1}{2} \alpha_{0} \alpha_{1} \\
\alpha_{0}-\frac{1}{2} \alpha_{1}^{2}
\end{array}\right), \quad B^{\sigma^{2}}\left(\alpha, \sigma^{2}\right)=\frac{3 \sigma^{2}}{4}\left(\frac{\alpha_{0}}{\alpha_{1}}-\alpha_{1}\right)
$$

Ces quantités étant explicites, nous réduirons l'erreur d'estimation en calculant les moyennes empiriques $\alpha_{N}^{d}, \alpha_{N}^{0}$ et $\sigma_{N}^{2}$ associées aux estimations partiellement débiaisées : $\widetilde{\widetilde{\alpha}}^{n}=\widetilde{\alpha}^{n}-$ $B^{d}\left(\widetilde{\alpha}^{n}\right) \delta, \quad \widehat{\widehat{\alpha}}^{n}=\widehat{\alpha}^{n}-B^{0}\left(\widehat{\alpha}^{n}\right) \delta$ et $\widehat{\widehat{\sigma}}_{n}^{2}=\widehat{\sigma}_{n}^{2}-B^{\sigma^{2}}\left(\widehat{\alpha}^{n}, \widehat{\sigma}_{n}^{2}\right) \delta$.

Le tableau 1 compare les biais réels $\alpha^{\delta}-\alpha, \widetilde{\alpha}^{\delta}-\alpha$ et $\sigma_{\delta}^{2}-\sigma^{2}$ à la partie principale des biais théoriques $B\left(\alpha, \sigma^{2}\right) \delta$. Le tableau 2 donne les estimations de $\alpha$ et de $\sigma^{2}$ comparées à leur limite théorique et aux estimations partiellement débiaisées. Le tableau 3 compare les variances empiriques aux parties principales des variances théoriques.

\begin{tabular}{|c|c|c|c|c|c|c|}
\hline$\delta$ & $\widetilde{\alpha}^{\delta}-\alpha$ & $B^{d}(\alpha) \delta$ & $\alpha^{\delta}-\alpha$ & $B^{0}(\alpha) \delta$ & $\sigma_{\delta}^{2}-\sigma^{2}$ & $B^{\sigma^{2}}\left(\alpha, \sigma^{2}\right) \delta$ \\
\hline 0.01 & -0.0297 & -0.03 & -0.0296 & -0.03 & -0.0173 & -0.0175 \\
& -0.0248 & -0.025 & -0.0125 & -0.0125 & & \\
\hline 0.05 & -0.1435 & -0.15 & -0.1407 & -0.15 & -0.0832 & -0.0875 \\
& -0.1213 & -0.125 & -0.0629 & -0.0625 & & \\
\hline 0.1 & -0.2749 & -0.3 & -0.2650 & -0.3 & -0.1587 & -0.175 \\
& -0.2356 & -0.25 & -0.1262 & -0.125 & & \\
\hline 0.5 & -1.0051 & -1.5 & -0.8956 & -1.5 & -0.5606 & -0.875 \\
& -0.9488 & -1.25 & -0.5953 & -0.625 & & \\
\hline
\end{tabular}

Table 1: Biais réels et parties principales des biais théoriques pour un $\operatorname{CAR}(2), \alpha_{0}=2, \alpha_{1}=3$ et $\sigma^{2}=1$.

Commentaire: les biais réels sont très proches de leurs parties principales pour $\delta=0.01$, $\delta=0.05$ et $\delta=0.1$. On constate la convergence des estimateurs vers leurs limites théoriques. L'estimation des variances reste correcte même pour des valeurs de $T$ relativement faibles, mais se dégrade rapidement lorsque $\delta$ croit. Les deux méthodes d'estimation de $\alpha$ sont proches l'une de l'autre. Le débiaisage partiel des estimations améliore globalement la précision de l'estimation. 


\begin{tabular}{|c|c|c|c|c|c|c|c|c|c|c|c|c|}
\hline$n$ & $\delta$ & $m_{N}^{d}(\alpha)$ & $\widetilde{\alpha}^{\delta}$ & $\alpha_{N}^{d}$ & $m_{N}^{0}(\alpha)$ & $\alpha^{\delta}$ & $\alpha_{N}^{0}$ & $\alpha$ & $m_{N}\left(\sigma^{2}\right)$ & $\sigma_{\delta}^{2}$ & $\overline{\sigma_{N}^{2}}$ & $\sigma^{2}$ \\
\hline \multirow{2}{*}{$\begin{array}{c}5000 \\
(\mathrm{~T}=50)\end{array}$} & 0.01 & 2.094 & 1.970 & 2.126 & 2.094 & 1.970 & 2.126 & 2 & 0.978 & 0.982 & 0.995 & 1 \\
\hline & & 3.024 & 2.975 & 3.050 & 3.032 & 2.987 & 3.044 & 3 & & & & \\
\hline \multirow{3}{*}{$(\mathrm{T}=250)$} & 0.05 & 1.871 & 1.856 & 2.006 & 1.874 & 1.859 & 2.012 & 2 & 0.917 & 0.916 & 0.996 & 1 \\
\hline & & 2.890 & 2.878 & 3.006 & 2.950 & 2.937 & 3.015 & 3 & & & & \\
\hline & 0.1 & 1.734 & 1.725 & 1.975 & 1.744 & 1.735 & 1.995 & 2 & 0.840 & 0.841 & 0.984 & 1 \\
\hline \multirow[t]{2}{*}{$(\mathrm{T}=500)$} & & 2.767 & 2.764 & 2.977 & 2.876 & 2.873 & 3.003 & 3 & & & & \\
\hline & 0.5 & 0.994 & 0.994 & 1.504 & 1.103 & 1.104 & 1.767 & 2 & 0.439 & 0.439 & 0.759 & 1 \\
\hline$(\mathrm{T}=2500)$ & & 2.051 & 2.051 & 2.607 & 2.403 & 2.404 & 2.917 & 3 & & & & \\
\hline \multirow{2}{*}{$\begin{array}{c}500 \\
(\mathrm{~T}=25)\end{array}$} & 0.05 & 2.124 & 1.856 & 2.282 & 2.126 & 1.859 & 2.288 & 2 & 0.903 & 0.916 & 0.982 & 1 \\
\hline & & 2.959 & 2.878 & 3.080 & 3.026 & 2.937 & 3.090 & 3 & & & & \\
\hline \multirow{3}{*}{$(\mathrm{T}=50)$} & 0.1 & 1.824 & 1.725 & 2.082 & 1.834 & 1.735 & 2.102 & 2 & 0.838 & 0.841 & 0.981 & 1 \\
\hline & & 2.813 & 2.764 & 3.033 & 2.917 & 2.873 & 3.046 & 3 & & & & \\
\hline & 0.5 & 1.006 & 0.994 & 1.528 & 1.116 & 1.104 & 1.790 & 2 & 0.436 & 0.439 & 0.756 & 1 \\
\hline$(\mathrm{T}=250)$ & & 2.061 & 2.051 & 2.633 & 2.412 & 2.404 & 2.930 & 3 & & & & \\
\hline
\end{tabular}

Table 2: Moyennes empiriques et estimations débiaisées d' un CAR(2) sur 200 répétitions, $\alpha_{0}=2, \alpha_{1}=3$ et $\sigma^{2}=1$.

\begin{tabular}{|c|c|c|c|c|c|c|}
\hline$n$ & $\delta$ & $V_{N}^{d}(\alpha)$ & $V_{N}^{0}(\alpha)$ & $\sigma^{2} \Gamma(0)^{-1}$ & $V_{N}\left(\sigma^{2}\right)$ & $\frac{9}{4} \sigma^{4}$ \\
\hline \multirow{2}{*}{$5000(\mathrm{~T}=50)$} & 0.01 & $\left(\begin{array}{ll}13.41 & -0.22 \\
-0.22 & 6.20\end{array}\right)$ & $\left(\begin{array}{cc}13.40 & -0.13 \\
-0.13 & 5.91\end{array}\right)$ & $\left(\begin{array}{ll}12 & 0 \\
0 & 6\end{array}\right)$ & 2.16 & 2.25 \\
\cline { 2 - 7 }$(\mathrm{T}=250)$ & 0.05 & $\left(\begin{array}{ll}10.32 & 0.92 \\
0.92 & 6.10\end{array}\right)$ & $\left(\begin{array}{cc}10.35 & 0.94 \\
0.94 & 5.49\end{array}\right)$ & $\left(\begin{array}{ll}12 & 0 \\
0 & 6\end{array}\right)$ & 1.62 & 2.25 \\
\cline { 2 - 8 }$(\mathrm{T}=500)$ & 0.1 & $\left(\begin{array}{ll}9.44 & 1.01 \\
1.01 & 6.63\end{array}\right)$ & $\left(\begin{array}{ll}9.64 & 1.42 \\
1.42 & 4.91\end{array}\right)$ & $\left(\begin{array}{ll}12 & 0 \\
0 & 6\end{array}\right)$ & 1.72 & 2.25 \\
\cline { 2 - 8 }$(\mathrm{T}=2500)$ & 0.5 & $\left(\begin{array}{ll}3.04 & 2.28 \\
2.28 & 9.72\end{array}\right)$ & $\left(\begin{array}{ll}4.02 & 1.90 \\
1.90 & 3.10\end{array}\right)$ & $\left(\begin{array}{ll}12 & 0 \\
0 & 6\end{array}\right)$ & 0.44 & 2.25 \\
\hline \multirow{2}{*}{$500(\mathrm{~T}=25)$} & 0.05 & $\left(\begin{array}{ll}14.27 & 1.03 \\
1.03 & 8.18\end{array}\right)$ & $\left(\begin{array}{ll}14.29 & 1.03 \\
1.03 & 6.82\end{array}\right)$ & $\left(\begin{array}{ll}12 & 0 \\
0 & 6\end{array}\right)$ & 2.31 & 2.25 \\
\cline { 2 - 8 }$(\mathrm{T}=50)$ & 0.1 & $\left(\begin{array}{ll}10.96 & 1.19 \\
1.19 & 5.96\end{array}\right)$ & $\left(\begin{array}{ll}11.12 & 1.37 \\
1.37 & 5.13\end{array}\right)$ & $\left(\begin{array}{ll}12 & 0 \\
0 & 6\end{array}\right)$ & 1.56 & 2.25 \\
\cline { 2 - 8 }$(\mathrm{T}=250)$ & 0.5 & $\left(\begin{array}{ll}3.88 & 3.96 \\
3.96 & 13.23\end{array}\right)$ & $\left(\begin{array}{ll}4.06 & 1.77 \\
1.77 & 13.52\end{array}\right)$ & $\left(\begin{array}{ll}12 & 0 \\
0 & 6\end{array}\right)$ & 0.48 & 2.25 \\
\hline
\end{tabular}

Table 3: Comparaison des variances empirique et théorique des estimateur d' un CAR(2) sur 200 répétitions, $\alpha_{0}=2, \alpha_{1}=3$ et $\sigma^{2}=1$. 


\section{References}

[1] Arató, M (1982) Linear Stochastic Systems with Constant Coefficients : a Statistical Approach.- Lecture Notes in control and information sciences, 45, Springer, Berlin.

[2] Bartlett, M.S. (1946) On the theoretical specification and sampling properties of autocorrelated time-series.- J. Roy. Statist. Soc., Ser. B, 8, 27-41.

[3] Bergstrom, (1976) Statistical inference in Continuous Time Series.- In Statistical inference in Continuous Time Economic Models., Ed. Bergstrom, North Holand.

[4] Brockwell, P. J. \& Hyndman, R. J. (1992) On continuous-time threshold autoregression.International Journal of Forecasting 8, North-Holland, 157-173.

[5] Doob, J. L. (1953) Stochastic Process.- Wiley, N.Y.

[6] Doukhan, P. (1995) Mixing : Properties and Examples.- Lecture Notes in Statistics 85, Springer-Verlag.

[7] Hyndman, R. J. (1993) Yule-Walker estimates for continuous-time autoregressive models. - Journal of Time Series Analysis, Vol. 14, No 3, 281-296.

[8] Phillips, A.W. (1959) The estimation of parameters in systems of stochastic differential equations.- Biometrika, 46, 67-76.

\section{A Démonstration du théorème 1 :}

$t \rightarrow X(t)$ est presque sûrement de classe $C^{p-1}$ sur $\mathbb{R}^{+}$. Par application de la formule de Taylor, on a, pour $t \geq 0$ et $h \geq 0$ :

$$
X(t+h)=\sum_{k=0}^{p-2} \frac{h^{k}}{k !} X^{(k)}(t)+\int_{t}^{t+h} \frac{(t+h-u)^{p-2}}{(p-2) !} X^{(p-1)}(u) d u
$$

Appliquant la formule de Ito à $f\left(v, X^{(j)}(v)\right)=\frac{(t+h-v)^{m}}{m !} X^{(j)}(v)$, on montre que :

$$
\begin{aligned}
\frac{(t+h-t-h)^{m}}{m !} X^{(j)}(t+h) & =\frac{(t+h-t)^{m}}{m !} X^{(j)}(t)-\int_{t}^{t+h} \frac{(t+h-v)^{m-1}}{(m-1) !} X^{(j)}(v) d v \\
& +\int_{t}^{t+h} \frac{(t+h-v)^{m}}{m !} d X^{(j)}(v)
\end{aligned}
$$

Donc, pour $m \geq 1$ et $j=0, p-1$ :

$$
\int_{t}^{t+h} \frac{(t+h-v)^{m-1}}{(m-1) !} X^{(j)}(v) d v=\frac{h^{m}}{m !} X^{(j)}(t)+\int_{t}^{t+h} \frac{(t+h-v)^{m}}{m !} d X^{(j)}(v)
$$

On a donc :

$$
\begin{aligned}
\int_{t}^{t+h} \frac{(t+h-v)^{p-2}}{(p-2) !} X^{(p-1)}(v) d v & =\frac{h^{p-1}}{(p-1) !} X^{(p-1)}(t)+\int_{t}^{t+h} \frac{(t+h-v)^{p-1}}{(p-1) !}\left(-\sum_{i=0}^{p-1} \alpha_{i} X^{(i)}(v)\right) d v \\
& +\int_{t}^{t+h} \frac{(t+h-v)^{p-1}}{(p-1) !} \sigma d W(v)
\end{aligned}
$$


et :

$X(t+h)=\sum_{k=0}^{p-1} \frac{h^{k}}{k !} X^{(k)}(t)+\int_{t}^{t+h} \frac{(t+h-v)^{p-1}}{(p-1) !}\left(-\sum_{i=0}^{p-1} \alpha_{i} X^{(i)}(v)\right) d v+\int_{t}^{t+h} \frac{(t+h-v)^{p-1}}{(p-1) !} \sigma d W(v)$

En appliquant (16) deux fois à $\int_{t}^{t+h} \frac{(t+h-v)^{p-1}}{(p-1) !}\left(-\sum_{i=0}^{p-1} \alpha_{i} X^{(i)}(v)\right) d v$, on obtient avec les notations introduites :

$$
\begin{aligned}
X(t+h) & =\sum_{k=0}^{p-1} \frac{h^{k}}{k !} X^{(k)}(t)-\frac{h^{p}}{p !} \sum_{i=0}^{p-1} \alpha_{i} X^{(i)}(t)+\frac{h^{p+1}}{(p+1) !} \sum_{i=0}^{p-1} \beta_{i} X^{(i)}(t) \\
& +\int_{t}^{t+h} \frac{(t+h-v)^{p-1}}{(p-1) !} \sigma d W(v)-\alpha_{p-1} \int_{t}^{t+h} \frac{(t+h-v)^{p}}{p !} \sigma d W(v) \\
& +\beta_{p-1} \int_{t}^{t+h} \frac{(t+h-v)^{p+1}}{(p+1) !} \sigma d W(v)+\int_{t}^{t+h} \frac{(t+h-v)^{p+1}}{(p+1) !}\left(\sum_{i=0}^{p-1} \gamma_{i} X^{(i)}(v)\right) d v
\end{aligned}
$$

Avec les $\left(a_{j}^{k}\right)$ définis précédemment, on a :

$$
\begin{aligned}
& \Delta^{(j)} X(t+j \delta)=\sum_{l=0}^{j} C_{j}^{l}(-1)^{j-l} X(t+l \delta) \\
& =\sum_{k=0}^{p-1} \frac{a_{j}^{k} \delta^{k}}{k !} X^{(k)}(t)-\frac{a_{j}^{p} \delta^{p}}{p !} \sum_{i=0}^{p-1} \alpha_{i} X^{(i)}(t)+\frac{a_{j}^{p+1} \delta^{p+1}}{(p+1) !} \sum_{i=0}^{p-1} \beta_{i} X^{(i)}(t) \\
& +\sum_{l=0}^{j} C_{j}^{l}(-1)^{j-l} \sum_{k=1}^{l} \int_{t+(k-1) \delta}^{t+k \delta}\left[\frac{(t+l \delta-v)^{p-1}}{(p-1) !}-\alpha_{p-1} \frac{(t+l \delta-v)^{p}}{p !}+\beta_{p-1} \frac{(t+l \delta-v)^{p+1}}{(p+1) !}\right] \sigma d W(v) \\
& +\sum_{l=0}^{j} C_{j}^{l}(-1)^{j-l} \sum_{k=1}^{l} \int_{t+(k-1) \delta}^{t+k \delta} \frac{(t+l \delta-v)^{p+1}}{(p+1) !}\left(\sum_{i=0}^{p-1} \gamma_{i} X^{(i)}(v)\right) d v
\end{aligned}
$$

De plus, si $k<j, a_{j}^{k}=0$. Le développement annoncé résulte de l'intervertion de l'ordre de sommation pour (17) et (18) :

$$
\begin{aligned}
& \Delta^{(j)} X(t+j \delta) \\
& =\sum_{k=j}^{p-1} \frac{a_{j}^{k} \delta^{k}}{k !} X^{(k)}(t)-\frac{a_{j}^{p}}{p !} \sum_{i=0}^{p-1} \alpha_{i} X^{(i)}(t)+\frac{a_{j}^{p+1}}{(p+1) !} \sum_{i=0}^{p-1} \beta_{i} X^{(i)}(t) \\
& +\sum_{k=1}^{j} \int_{t+(k-1) \delta}^{t+k \delta} \sum_{l=k}^{j} C_{j}^{l}(-1)^{j-l}\left[\frac{(t+l \delta-v)^{p-1}}{(p-1) !}-\alpha_{p-1} \frac{(t+l \delta-v)^{p}}{p !}+\beta_{p-1} \frac{(t+l \delta-v)^{p+1}}{(p+1) !}\right] \sigma d W(v) \\
& +\sum_{k=1}^{j} \int_{t+(k-1) \delta}^{t+k \delta} \sum_{l=k}^{j} C_{j}^{l}(-1)^{j-l} \frac{(t+l \delta-v)^{p+1}}{(p+1) !}\left(\sum_{i=0}^{p-1} \gamma_{i} X^{(i)}(v)\right) d v
\end{aligned}
$$




\section{B Démonstration du Théorème 4}

Loi asymptotique de $\widehat{\alpha}^{n}: \widehat{\alpha}^{n}-\alpha^{\delta}=\left[\widehat{\Gamma}^{n}\right]^{-1}\left(\widehat{\gamma}^{n}+\widehat{\Gamma}^{n} \widehat{\alpha}^{\delta}\right)$ est centré. Notons, pour $i=0, p-1, l=0, p-1, \beta_{l}^{i}=\alpha_{l}^{\delta}$, et pour $i=0, p-2, \beta_{p}^{i}=1$ et $\beta_{p}^{p-1}=c(p)^{-1}$. On a : $\widehat{\gamma}^{n}+\widehat{\Gamma}^{n} \widehat{\alpha}^{\delta}={ }^{t}\left(\sum_{l=0}^{p} \beta_{l}^{0} \widehat{D}_{0, l}^{n}, \cdots, \sum_{l=0}^{p} \beta_{l}^{p-1} \widehat{D}_{p-1, l}^{n}\right)$. L'application du théorème de limite centrale donne :

$$
\sqrt{n \delta}\left(\widehat{\gamma}^{n}+\widehat{\Gamma}^{n} \alpha^{\delta}\right) \stackrel{\mathcal{D}(\mathbb{P})}{\rightarrow} \mathcal{N}_{p}\left(0, M_{\theta}\right), \quad M_{\theta}=\left(M_{i, j}\right)_{i, j=0, p-1}
$$

Notons : $A_{i} X(t)=\sum_{l=0}^{p} \delta^{p-l} \beta_{l}^{i} \Delta^{(l)} X(t+l \delta)$. Les coefficients de $M_{\theta}$ sont, pour $i, j=0, p-1$ :

$$
\begin{aligned}
M_{i, j} & =\frac{1}{\delta^{2 p-1+i+j}} \sum_{k=0}^{+\infty} \mathbb{E}\left[\Delta^{(i)} X(i \delta) \Delta^{(j)} X(j \delta+k \delta) A_{i}(0) A_{j}(k \delta)\right] \\
& +\frac{1}{\delta^{2 p-1+i+j}} \sum_{k=1}^{+\infty} \mathbb{E}\left[\Delta^{(j)} X(j \delta) \Delta^{(i)} X(i \delta+k \delta) A_{i} X(k \delta) A_{j} X(0)\right]
\end{aligned}
$$

Le processus $X$ étant gaussien et centré, on applique la propriété $\mathbb{E}(X Y Z T)=\mathbb{E}(X Y) \mathbb{E}(Z T)+$ $\mathbb{E}(X Z) \mathbb{E}(Y T)+\mathbb{E}(X T) \mathbb{E}(Y Z)$ avec $X=\Delta^{(i)} X, Y=\Delta^{(j)} X, Z=A_{i} X$ et $T=A_{j} X$, puis (10). $M_{i, j}$ se décompose alors comme suit :

$$
\begin{aligned}
\delta^{2 p-1+i+j} M_{i, j} & =\sum_{k=0}^{+\infty}(-1)^{i} \Delta^{(i+j)} r(j \delta+k \delta)\left(\sum_{l=0}^{p} \delta^{p-l} \beta_{l}^{i}(-1)^{l} \sum_{q=0}^{p} \delta^{p-q} \beta_{q}^{j} \Delta^{(l+q)} r(q \delta+k \delta)\right) \\
& +\sum_{k=0}^{+\infty}\left(\sum_{l=0}^{p} \delta^{p-l} \beta_{l}^{i}(-1)^{l} \Delta^{(l+j)} r(j \delta+k \delta)\right)\left(\sum_{q=0}^{p} \delta^{p-q} \beta_{q}^{j}(-1)^{i} \Delta^{(q+i)} r(q \delta+k \delta)\right) \\
& +\sum_{k=0}^{+\infty}\left(\sum_{l=0}^{p} \delta^{p-l} \beta_{l}^{i}(-1)^{i} \Delta^{(l+i)} r(l \delta)\right)\left(\sum_{q=0}^{p} \delta^{p-q} \beta_{q}^{j}(-1)^{j} \Delta^{(q+j)} r(q \delta)\right) \\
& +\sum_{k=1}^{+\infty}(-1)^{j} \Delta^{(i+j)} r(i \delta+k \delta)\left(\sum_{q=0}^{p} \delta^{p-q} \beta_{q}^{j}(-1)^{q} \sum_{l=0}^{p} \delta^{p-l} \beta_{l}^{i} \Delta^{(l+q)} r(l \delta+k \delta)\right) \\
& +\sum_{k=1}^{+\infty}\left(\sum_{l=0}^{p} \delta^{p-l} \beta_{l}^{i}(-1)^{j} \Delta^{(l+j)} r(l \delta+k \delta)\right)\left(\sum_{q=0}^{p} \delta^{p-q} \beta_{q}^{j}(-1)^{q} \Delta^{(q+i)} r(i \delta+k \delta)\right) \\
& +\sum_{k=1}^{+\infty}\left(\sum_{l=0}^{p} \delta^{p-l} \beta_{l}^{i}(-1)^{i} \Delta^{(l+i)} r(l \delta)\right)\left(\sum_{q=0}^{p} \delta^{p-q} \beta_{q}^{j}(-1)^{j} \Delta^{(q+j)} r(q \delta)\right)
\end{aligned}
$$

Par définition de $\alpha^{\delta},(21)$ et (24) sont nuls. Analysons (19) : utilisant (11), on a pour $k \geq p$,

$$
\begin{aligned}
& \Delta^{(i+j)} r(j \delta+k \delta)\left(\sum_{l=0}^{p} \delta^{p-l} \beta_{l}^{i}(-1)^{l} \sum_{q=0}^{p} \delta^{p-q} \beta_{q}^{j} \Delta^{(l+q)} r(q \delta+k \delta)\right) \\
& =\delta^{i+j+2 p} r^{(i+j)}(k \delta)\left(\sum_{l=0}^{p} \beta_{l}^{i}(-1)^{l} \sum_{q=0}^{p} \beta_{q}^{j} r^{(l+q)}(k \delta)\right)+\delta^{i+j+2 p+1} R_{1}(k \delta)
\end{aligned}
$$


Par définition de $\beta_{q}^{j}$, on a :

$$
\begin{aligned}
\sum_{l=0}^{p} \beta_{l}^{i}(-1)^{l} \sum_{q=0}^{p} \beta_{q}^{j} r^{(l+q)}(k \delta) & =\sum_{l=0}^{p} \beta_{l}^{i}(-1)^{l}\left[\beta_{p}^{j} r^{(p+l)}(k \delta)+\sum_{q=0}^{p-1} \alpha_{q}^{\delta} r^{(l+q)}(k \delta)\right] \\
& =\sum_{l=0}^{p} \beta_{l}^{i}(-1)^{l}\left[\beta_{p}^{j} r^{(p+l)}(k \delta)+\sum_{q=0}^{p-1} \alpha_{q} r^{(l+q)}(k \delta)\right] \\
& +\sum_{l=0}^{p} \beta_{l}^{i}(-1)^{l} \sum_{q=0}^{p-1}\left(\alpha_{q}^{\delta}-\alpha_{q}\right) r^{(l+q)}(k \delta)
\end{aligned}
$$

Puisque $\alpha^{\delta}-\alpha=O(\delta)$ et que $r$ satisfait les équations de Yule-Walker, on obtient alors :

$$
\sum_{l=0}^{p} \beta_{l}^{i}(-1)^{l} \sum_{q=0}^{p} \beta_{q}^{j} r^{(l+q)}(k \delta)=\sum_{l=0}^{p} \beta_{l}^{i}(-1)^{l}\left(\beta_{p}^{j}-1\right) r^{(p+l)}(k \delta)+\delta R_{2}(k \delta)
$$

On a ainsi :

$$
\begin{aligned}
& \sum_{k=p}^{+\infty}(-1)^{i} \Delta^{(i+j)} r(j \delta+k \delta)\left(\sum_{l=0}^{p} \delta^{p-l} \beta_{l}^{i}(-1)^{l} \sum_{q=0}^{p} \delta^{p-q} \beta_{q}^{j} \Delta^{(l+q)} r(q \delta+k \delta)\right) \\
& =\delta^{i+j+2 p} \sum_{k=0}^{+\infty}(-1)^{i} r^{(i+j)}(k \delta)\left[\sum_{l=0}^{p} \beta_{l}^{i}(-1)^{l}\left(\beta_{p}^{j}-1\right) r^{(p+l)}(k \delta)\right]+\delta^{i+j+2 p+1} \sum_{k=0}^{+\infty} R(k \delta)
\end{aligned}
$$

où $R$ est à décroissance exponentielle. Pour $\delta$ proche de 0 et compte tenu de la régularité de $r$ et de ses dérivées, on obtient :

$$
\begin{aligned}
& \sum_{k=p}^{+\infty}(-1)^{i} \Delta^{(i+j)} r(j \delta+k \delta)\left(\sum_{l=0}^{p} \delta^{p-l} \beta_{l}^{i}(-1)^{l} \sum_{q=0}^{p} \delta^{p-q} \beta_{q}^{j} \Delta^{(l+q)} r(q \delta+k \delta)\right) \\
& =\delta^{i+j+2 p-1} \int_{v=0}^{+\infty}(-1)^{i} r^{(i+j)}(v)\left[\sum_{l=0}^{p} \beta_{l}^{i}(-1)^{l}\left(\beta_{p}^{j}-1\right) r^{(p+l)}(v)\right] d v+O\left(\delta^{i+j+2 p}\right)
\end{aligned}
$$

Donc :

$$
\begin{aligned}
(19) & =\delta^{i+j+2 p-1} \int_{v=0}^{+\infty}(-1)^{i} r^{(i+j)}(v)\left[\sum_{l=0}^{p} \beta_{l}^{i}(-1)^{l}\left(\beta_{p}^{j}-1\right) r^{(p+l)}(v)\right] d v+O\left(\delta^{i+j+2 p}\right) \\
& +\sum_{k=0}^{p-1}(-1)^{i} \Delta^{(i+j)} r(j \delta+k \delta)\left(\sum_{l=0}^{p} \delta^{p-l} \beta_{l}^{i}(-1)^{l} \sum_{q=0}^{p} \delta^{p-q} \beta_{q}^{j} \Delta^{(l+q)} r(q \delta+k \delta)\right)
\end{aligned}
$$

On montre de manière similaire que :

$$
\begin{aligned}
(22) & =\delta^{i+j+2 p-1} \int_{v=0}^{+\infty}(-1)^{j} r^{(i+j)}(v)\left[\sum_{q=0}^{p} \beta_{q}^{j}(-1)^{q}\left(\beta_{p}^{i}-1\right) r^{(p+l)}(v)\right] d v+O\left(\delta^{i+j+2 p}\right) \\
& +\sum_{k=1}^{p-1}(-1)^{j} \Delta^{(i+j)} r(i \delta+k \delta)\left(\sum_{q=0}^{p} \delta^{p-l} \beta_{q}^{j}(-1)^{q} \sum_{l=0}^{p} \delta^{p-q} \beta_{l}^{i} \Delta^{(l+q)} r(l \delta+k \delta)\right)
\end{aligned}
$$


$(20)=\delta^{i+j+2 p-1} \int_{v=0}^{+\infty}(-1)^{i}\left(\beta_{p}^{j}-1\right) r^{(i+p)}(v)\left(\sum_{l=0}^{p} \beta_{l}^{i}(-1)^{l} r^{(l+j)}(v)\right) d v+O\left(\delta^{i+j+2 p}\right)$

$(23)=\delta^{i+j+2 p-1} \int_{v=0}^{+\infty}(-1)^{j}\left(\beta_{p}^{i}-1\right) r^{(j+p)}(v)\left(\sum_{q=0}^{p} \beta_{q}^{j}(-1)^{q} r^{(i+q)}(v)\right) d v+O\left(\delta^{i+j+2 p}\right)$

D'après l'équation (10) du théorème 2 , on a :

$$
\begin{aligned}
& \sum_{k=1}^{p-1}(-1)^{j} \Delta^{(i+j)} r(i \delta+k \delta)\left(\sum_{q=0}^{p} \delta^{p-l} \beta_{q}^{j}(-1)^{q} \sum_{l=0}^{p} \delta^{p-q} \beta_{l}^{i} \Delta^{(l+q)} r(l \delta+k \delta)\right) \\
& =\sum_{k=1}^{p-1}(-1)^{i} \Delta^{(i+j)} r(j \delta-k \delta)\left(\sum_{l=0}^{p} \delta^{p-l} \beta_{l}^{i}(-1)^{l} \sum_{q=0}^{p} \delta^{p-q} \beta_{q}^{j} \Delta^{(l+q)} r(q \delta-k \delta)\right)
\end{aligned}
$$

$M_{i, j}$ s'écrit donc :

$$
\begin{aligned}
M_{i, j} & =\int_{v=0}^{+\infty}(-1)^{i} r^{(i+j)}(v)\left[\sum_{l=0}^{p} \beta_{l}^{i}(-1)^{l}\left(\beta_{p}^{j}-1\right) r^{(p+l)}(v)\right] d v \\
& +\int_{v=0}^{+\infty}(-1)^{j} r^{(i+j)}(v)\left[\sum_{q=0}^{p} \beta_{q}^{j}(-1)^{q}\left(\beta_{p}^{i}-1\right) r^{(p+l)}(v)\right] d v \\
& +\int_{v=0}^{+\infty}(-1)^{i}\left(\beta_{p}^{j}-1\right) r^{(i+p)}(v)\left(\sum_{l=0}^{p} \beta_{l}^{i}(-1)^{l} r^{(l+j)}(v)\right) d v \\
& +\int_{v=0}^{+\infty}(-1)^{j}\left(\beta_{p}^{i}-1\right) r^{(j+p)}(v)\left(\sum_{q=0}^{p} \beta_{q}^{j}(-1)^{q} r^{(i+q)}(v)\right) d v+O(\delta) \\
& +\frac{1}{\delta^{i+j+2 p-1}} \sum_{k=-(p-1)}^{p-1}(-1)^{i} \Delta^{(i+j)} r(j \delta+k \delta)\left(\sum_{l=0}^{p} \delta^{p-l} \beta_{l}^{i}(-1)^{l} \sum_{q=0}^{p} \delta^{p-q} \beta_{q}^{j} \Delta^{(l+q)} r(q \delta+k \delta)\right)
\end{aligned}
$$

Or, utilisant (12), on montre que :

$$
\begin{aligned}
& \sum_{k=-(p-1)}^{p-1}(-1)^{i} \Delta^{(i+j)} r(j \delta+k \delta)\left(\sum_{l=0}^{p} \delta^{p-l} \beta_{l}^{i}(-1)^{l} \sum_{q=0}^{p} \delta^{p-q} \beta_{q}^{j} \Delta^{(l+q)} r(q \delta+k \delta)\right) \\
= & \beta_{p}^{i} \beta_{p}^{j}(-1)^{p} \sum_{k=-(p-1)}^{p-1}(-1)^{i} \Delta^{(i+j)} r(j \delta+k \delta) \Delta^{(2 p)} r(p \delta+k \delta)+O\left(\delta^{i+j+2 p}\right) \\
= & \beta_{p}^{i} \beta_{p}^{j}(-1)^{p} \delta^{i+j}(-1)^{i} r^{(i+j)}(0) \sum_{k=-(p-1)}^{p-1} \Delta^{(2 p)} r(p \delta+k \delta)+O\left(\delta^{i+j+2 p}\right)
\end{aligned}
$$

avec :

$$
\begin{aligned}
\sum_{k=-(p-1)}^{p-1} \Delta^{(2 p)} r(p \delta+k \delta) & =\sum_{k=-(p-1)}^{p-1}\left[\Delta^{(2 p-1)} r(p \delta+k \delta)-\Delta^{(2 p-1)} r(p \delta+(k-1) \delta)\right] \\
& =\Delta^{(2 p-1)} r((2 p-1) \delta)-\Delta^{(2 p-1)} r(0)=2 \delta^{2 p-1}(-1)^{p} r^{(2 p-1)}\left(0^{+}\right)+O\left(\delta^{2 p}\right)
\end{aligned}
$$


D'où, l'expression finale de $M_{i, j}$ :

$$
\begin{aligned}
M_{i, j} & =\int_{v=0}^{+\infty}(-1)^{i} r^{(i+j)}(v)\left[\sum_{l=0}^{p} \beta_{l}^{i}(-1)^{l}\left(\beta_{p}^{j}-1\right) r^{(p+l)}(v)\right] d v \\
& +\int_{v=0}^{+\infty}(-1)^{j} r^{(i+j)}(v)\left[\sum_{q=0}^{p} \beta_{q}^{j}(-1)^{q}\left(\beta_{p}^{i}-1\right) r^{(p+l)}(v)\right] d v \\
& +\int_{v=0}^{+\infty}(-1)^{i}\left(\beta_{p}^{j}-1\right) r^{(i+p)}(v)\left(\sum_{l=0}^{p} \beta_{l}^{i}(-1)^{l} r^{(l+j)}(v)\right) d v \\
& +\int_{v=0}^{+\infty}(-1)^{j}\left(\beta_{p}^{i}-1\right) r^{(j+p)}(v)\left(\sum_{q=0}^{p} \beta_{q}^{j}(-1)^{q} r^{(i+q)}(v)\right) d v \\
& +2 \beta_{p}^{i} \beta_{p}^{j}(-1)^{i} r^{(i+j)}(0)(-1)^{p} r^{(2 p-1)}\left(0^{+}\right)+O(\delta)
\end{aligned}
$$

Pour $i=0, p-2$ et $j=0, p-2$, on a $\beta_{p}^{i}=\beta_{p}^{j}=1$. Donc :

$$
M_{i, j}=2(-1)^{i} r^{(i+j)}(0)(-1)^{p} r^{(2 p-1)}\left(0^{+}\right)+O(\delta)
$$

Pour $i=0, p-2$ et $j=p-1$, on a $\beta_{p}^{i}=1$ et $\beta_{p}^{j}=c(p)^{-1}$. Ainsi :

$$
\begin{aligned}
M_{i, p-1} & =\left(\frac{1}{c(p)}-1\right) \int_{v=0}^{+\infty}(-1)^{i} r^{(i+p-1)}(v)\left[\sum_{l=0}^{p} \beta_{l}^{i}(-1)^{l} r^{(p+l)}(v)\right] d v \\
& +\left(\frac{1}{c(p)}-1\right) \int_{v=0}^{+\infty}(-1)^{i} r^{(i+p)}(v)\left[\sum_{l=0}^{p} \beta_{l}^{i}(-1)^{l} r^{(l+p-1)}(v)\right] d v \\
& +\frac{2}{c(p)}(-1)^{i} r^{(i+p-1)}(0)(-1)^{p} r^{(2 p-1)}\left(0^{+}\right)+O(\delta) \\
& =\left(\frac{1}{c(p)}-1\right)(-1)^{i} \sum_{l=0}^{p} \beta_{l}^{i}(-1)^{l} \int_{v=0}^{+\infty} r^{(i+p-1)}(v) r^{(p+l)}(v)+r^{(i+p)}(v) r^{(l+p-1)}(v) d v \\
& +\frac{2}{c(p)}(-1)^{i} r^{(i+p-1)}(0)(-1)^{p} r^{(2 p-1)}\left(0^{+}\right)+O(\delta) \\
& =\left(\frac{1}{c(p)}-1\right)(-1)^{i} \sum_{l=0}^{p} \beta_{l}^{i}(-1)^{l}\left[r^{(i+p-1)}(v) r^{(l+p-1)}(v)\right]_{0}^{+\infty} \\
& +\frac{2}{c(p)}(-1)^{i} r^{(i+p-1)}(0)(-1)^{p} r^{(2 p-1)}\left(0^{+}\right)+O(\delta)
\end{aligned}
$$

Utilisant les équations de Yule-Walker en 0, on obtient :

$$
\begin{aligned}
M_{i, p-1} & =-\left(\frac{1}{c(p)}-1\right)(-1)^{i} r^{(i+p-1)}(0)\left[(-1)^{p} r^{(2 p-1)}\left(0^{+}\right)-(-1)^{p} r^{(2 p-1)}\left(0^{-}\right)\right] \\
& +\frac{2}{c(p)}(-1)^{i} r^{(i+p-1)}(0)(-1)^{p} r^{(2 p-1)}\left(0^{+}\right)+O(\delta) \\
& =\left[-2\left(\frac{1}{c(p)}-1\right)+\frac{2}{c(p)}\right](-1)^{i} r^{(i+p-1)}(0)(-1)^{p} r^{(2 p-1)}\left(0^{+}\right)+O(\delta) \\
& =2(-1)^{i} r^{(i+p-1)}(0)(-1)^{p} r^{(2 p-1)}\left(0^{+}\right)+O(\delta)
\end{aligned}
$$


On montre de manière similaire que :

$$
\begin{array}{ll}
\forall j=0, p-2, \quad M_{p-1, j}=2(-1)^{j} r^{(p-1+j)}(0)(-1)^{p} r^{(2 p-1)}\left(0^{+}\right)+O(\delta) \\
& M_{p-1, p-1}=2(-1)^{p-1} r^{(2 p-2)}(0)(-1)^{p} r^{(2 p-1)}\left(0^{+}\right)+O(\delta)
\end{array}
$$

Etude de la loi asymptotique de $\widehat{\sigma}_{n}^{2}$ : elle repose sur des techniques similaires.

$$
\sqrt{n}\left(\widehat{D}_{p-1, p}^{n}-\mathbb{E}\left[\widehat{D}_{p-1, p}^{n}\right]\right) \stackrel{\mathcal{D}(\mathbb{P})}{\longrightarrow} \mathcal{N}\left(0, C_{\theta}\right)
$$

où $C_{\theta}$ est donnée par :

$$
\begin{aligned}
C_{\theta} & =\frac{(-1)^{2 p-1}}{\delta^{4 p-2}} \sum_{k=-\infty}^{+\infty} \Delta^{(2 p-2)} r((p-1) \delta+k \delta) \Delta^{(2 p)} r(p \delta+k \delta) \\
& +\frac{(-1)^{2 p-1}}{\delta^{4 p-2}} \sum_{k=-\infty}^{+\infty} \Delta^{(2 p-1)} r((p-1) \delta+k \delta) \Delta^{(2 p-1)} r(p \delta+k \delta)
\end{aligned}
$$

Or on montre que :

$$
C_{\theta}=\frac{(-1)^{2 p}}{2 \delta^{4 p-2}} \sum_{k=-\infty}^{+\infty}\left[\Delta^{(2 p)} r(p \delta+k \delta)\right]^{2}
$$

avec, par application du théorème 2 :

$$
\frac{(-1)^{2 p}}{2 \delta^{4 p-2}} \sum_{k=-\infty}^{-p}\left[\Delta^{(2 p)} r(p \delta+k \delta)\right]^{2}=O(\delta), \quad \frac{(-1)^{2 p}}{2 \delta^{4 p-2}} \sum_{k=p}^{+\infty}\left[\Delta^{(2 p)} r(p \delta+k \delta)\right]^{2}=O(\delta)
$$

On a alors :

$$
C_{\theta}=\frac{(-1)^{2 p}}{2 \delta^{4 p-2}} \sum_{k=-(p-1)}^{p-1}\left[\Delta^{(2 p)} r(p \delta+k \delta)\right]^{2}+O(\delta)=\frac{1}{2 \delta^{4 p-2}} \sum_{k=1}^{2 p-1}\left[\Delta^{(2 p)} r(k \delta)\right]^{2}+O(\delta)
$$

Utilisant (12), on obtient :

$C_{\theta}=\frac{1}{2 \delta^{4 p-2}} \sum_{k=1}^{2 p-1}\left[\delta^{2 p-1} d_{2 p, k} r^{(2 p-1)}\left(0^{+}\right)\right]^{2}+O(\delta)=\frac{\sigma^{4}}{2} \sum_{k=1}^{2 p-1}\left[\sum_{l=0}^{k} C_{2 p}^{l}(-1)^{l} \frac{(k-l)^{2 p-1}}{(2 p-1) !}\right]^{2}+O(\delta)$

Enfin : $K_{\theta}=\left(\frac{-2}{c(p)}\right)^{2} C_{\theta} . \square$ 